\title{
Multi-level hypothesis testing for populations of heterogeneous networks ${ }^{1}$
}

\author{
Guilherme Gomes \\ Department of Statistics \\ Purdue University \\ West Lafayette, USA \\ gomesg@purdue.edu
}

\author{
Vinayak Rao \\ Department of Statistics \\ Purdue University \\ West Lafayette, USA \\ varao@purdue.edu
}

\author{
Jennifer Neville \\ Department of Computer Science \\ Purdue University \\ West Lafayette, USA \\ neville@cs.purdue.edu
}

\begin{abstract}
In this work, we consider hypothesis testing and anomaly detection on datasets where each observation is a weighted network. Examples of such data include brain connectivity networks from fMRI flow data, or word co-occurrence counts for populations of individuals. Current approaches to hypothesis testing for weighted networks typically requires thresholding the edge-weights, to transform the data to binary networks. This results in a loss of information, and outcomes are sensitivity to choice of threshold levels. Our work avoids this, and we consider weighted-graph observations in two situations, 1) where each graph belongs to one of two populations, and 2) where entities belong to one of two populations, with each entity possessing multiple graphs (indexed e.g. by time). Specifically, we propose a hierarchical Bayesian hypothesis testing framework that models each population with a mixture of latent space models for weighted networks, and then tests populations of networks for differences in distribution over components. Our framework is capable of population-level, entity-specific, as well as edge-specific hypothesis testing. We apply it to synthetic data and three real-world datasets: two social media datasets involving word co-occurrences from discussions on Twitter of the political unrest in Brazil, and on Instagram concerning Attention Deficit Hyperactivity Disorder (ADHD) and depression drugs, and one medical dataset involving fMRI brain-scans of human subjects. The results show that our proposed method has lower Type I error and higher statistical power compared to alternatives that need to threshold the edge weights. Moreover, they show our proposed method is better suited to deal with highly heterogeneous datasets.
\end{abstract}

\section{INTRODUCTION}

We consider the problem of graph-based hypothesis testing, which tests whether two sets of graph-valued observation samples are drawn from the same distribution. This is a topic of growing interest [2]-[5]; however, there are only a few studies where the observations are weighted graphs. In this work, we address this gap, considering the problem of hypothesis-testing for replicated weighted graph-valued data. This is a challenging problem, since the average and atypical behavior of a sample of networks is difficult to characterize.

Figure 1 illustrates two example domains with populations of weighted graphs. The top row illustrates the word cooccurrence networks of two Twitter users in Brazil, one that

\footnotetext{
${ }^{1}$ This is the extended version of 1 .
}

is pro-government and one that is anti-government. Here the entity corresponds to a user on social media, the nodes in the graph are vocabulary words, and the edges weights reflect co-occurrences of words in the posts of the users. The bottom row illustrates brain connectivity networks of two individuals, one female and one male. Here the entity corresponds to an individual, the nodes in the graph are brain regions, and the edges weights represent functional connectivity strength as measured by functional magnetic resonance imaging (fMRI). In both cases, we would like to investigate how populations and entities differ. For example, whether brain activity differs with respect to sex or whether word usage differs with respect to political views.

There has been recent work on graph-based hypothesis testing (see [6] for a good survey). However, much of the work has focused on testing subgraphs within a larger graph (e.g., [7]), or on one-sample tests comparing a single graph to a null model (e.g., [8]). Work focusing on populations of graphs has received considerably less attention and falls into one of two categories: that of [9], which introduces a geometric characterization of the network using the so-called Fréchet mean, and that of [10], who proposed a Bayesian latent-variable model for unweighted graphs. We focus on the latter, which allows us to bring the powerful machinery of probabilistic hierarchical modeling to the table, allowing noisiness and missingness, and providing interpretable confidence scores. Unfortunately, existing work along this second direction is limited to modeling binary graphs, so that in practice, a threshold must be used to transform counts or continuous weights to $0 / 1$ values. Such a thresholding operation discards valuable information about the strength of the edge-weights, and can also exhibit sensitivity towards the choice of threshold. Too small a threshold can result in a graph that is too dense, and too large, too sparse. Often, there does not even exist a single appropriate threshold across the entire graph.

In this paper, we address the issues of previous work and develop a hypothesis testing framework that facilitates testing over graphs populations with edge-weights, which can follow any parametric distribution. Specifically, we propose a Bayesian hypothesis testing framework that uses a mixture of latent space models for weighted networks to 


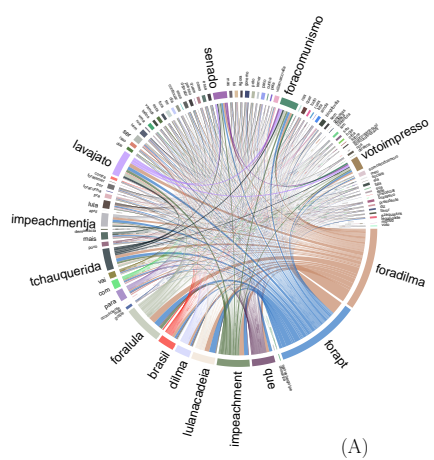

(A)

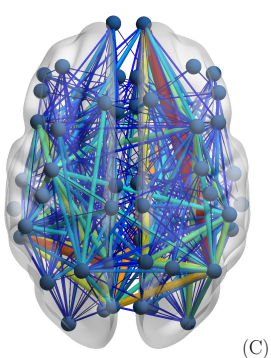

(C)

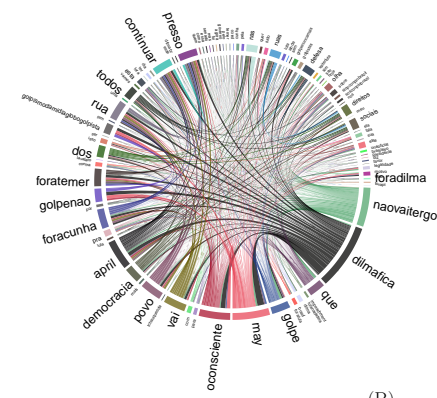

Figure 1: Top row: word connectivity networks for two Brazilian Twitter users, (A) from the pro-government side and (B) from the anti-government side. Bottom row: brain connectivity network for two individuals, (C) female and (D) male.

test for population-differences. Our framework is capable of population-level, entity-specific as well as edge-specific hypothesis testing. We consider testing in two broad scenarios:

1) When all observations from the same population follow the same distribution, we can ask: Are the population distributions identical?

2) When all entities from the same population follow the same distribution, we can ask: Are the population distributions identical? Now, every entity has an associated set of graph-valued observations which are identically distributed, but are not exchangeable across entities.

Observe the first case is equivalent to the second when each entity has only one associated graph; however the latter allows heterogeneity among entities in the same population. For instance an entity in a population that is politically conservative might frame an issue they discuss from an economic perspective, while another entity in a population that is politically liberal might focus on the social aspects of the same issue. [11] proposed a strongly parametric timevarying framework to handle this important situation, our approach is significantly more flexible.

We apply our testing framework to problems from the types of domains summarized in Figure 1 . First, we look at word co-occurrence network data from Twitter (on the political crisis in Brazil), as well as Instagram (on side effects of Adderall and Ritalin usage for Attention Deficit Hyperactivity Disorder [12]). In both datasets, we investigate how populations and entities differ based on the way they communicate-specially in the manner in which the usage of pairs of key words differs between groups. Standard methods such as unigram mixture models, latent Dirichlet allocation (LDA) [13] or N-gram language models [14], which are based just on word-frequency, do not capture the kind of contextual information we are interested in. While these methods can identify words that 'belong' to different groups, in our scenario there is a strong overlap in key words across groups, and such models will fail to differentiate between groups which share common themes and vocabularies. Second, we used functional magnetic resonance imaging (fMRI) data, to investigate how brain activity differs across populations like sex, age and personality traits like extroversion, conscientiousness and creativity. In both tasks, we show that graph-structure as well as graph weights are crucial to performance, and that we outperform baselines like latent Dirichlet allocation (LDA) [13], N-gram language models [14], as well as thresholding methods like [10].

Contributions: Our contribution is a multi-level statistical hypothesis testing framework for populations of weighted networks, concerning both the overall graph distributions, as well as two types of local hypotheses: entity-specific and edge-specific. The latter are important since a population might have networks, or a network edges, that are statistically different, and that might escape detection by a global test. Our hierarchical Bayesian mixed membership model allows statistical information to be shared across groups, increasing accuracy of hypothesis tests without loss of statistical power. This allows practitioners to evaluate anomalies in a principled manner, using statistical significance. Notably, our framework is more robust than previous methods developed for binary graphs, which require thresholding of weighted data before application.

\section{THE MODEL}

We are given a set $\mathbf{A}$ of undirected graphs, with graph $A_{n t}$ belonging to entity $n$ at index $t$ (refered to as 'time'). Here $n \in[1, \ldots, N]$ and $t \in\left[1, \ldots, T_{n}\right]$, with $A_{n t}[i, j]$ giving the link strength between vertices $i$ and $j$ of entity $n$ at time $t(i, j \in[1, \ldots, V])$. We also observe population information $y_{n} \in[1, \ldots, G]$ for each entity. For instance, each network might represent word co-occurrences in a user's social media messages over some time period, while the population might indicate whether the user's political leanings are 'Liberal' or 'Conservative'.

Underlying our testing framework is a probabilistic model which we now outline. We assume each observation $A_{n t}$ comes from one of $H$ clusters or mixture components, with cluster $h$ having parameter $\boldsymbol{\theta}^{(h)}$. Each cluster has a distribution over graphs which we write as $F\left(\boldsymbol{\theta}^{(h)}\right)$ (we specify $\boldsymbol{\theta}^{(h)}$ 
and $F$ in the next paragraph). Clusters and cluster parameters are shared across populations, however each population $y$ has its own Dirichlet-distributed probability over clusters, $\boldsymbol{\beta}_{y}$. At a high-level ours is a Bayesian hypothesis-testing approach which tests whether the $\boldsymbol{\beta}_{y}$ 's are identical across populations. For the case of two populations, we place equal a priori probability on the null hypothesis $H_{0}: \boldsymbol{\beta}_{1}=\boldsymbol{\beta}_{2}$ and the alternative $H_{1}: \boldsymbol{\beta}_{1} \neq \boldsymbol{\beta}_{2}$. Using the machinery of Bayesian inference, we evaluate the posterior probabilities of the two hypotheses given observations, and reject the null if its probability $P\left(H_{0} \mid-\right)$ is less than some specified threshold (e.g., 0.05 or 0.1 ).

We now describe the cluster-specific distribution over graphs, $F\left(\boldsymbol{\theta}^{(h)}\right)$. For cluster $h, \boldsymbol{\theta}^{(h)}$ is a $V \times V$ matrix, whose $(i j)$ th element parametrizes the probability of the weight on the edge between nodes $i$ and $j$. In our applications, we looked at count-valued edges, and so assumed $F\left(\boldsymbol{\theta}^{(h)}\right)$ to be Binomial or Poisson distributed with parameter $\boldsymbol{\theta}^{(h)}[i, j]$ on edge $(i, j)$. We define $\boldsymbol{\theta}^{(h)}=f\left(\boldsymbol{S}^{(h)}\right)$ where $f(\cdot)$ is some link function (e.g. the logistic or exponential function to ensure nonnegativity), and constrain $\boldsymbol{S}$ using a low-rank factorization scheme $\boldsymbol{S}^{(h)}=\mathbf{X}^{(h)} \mathbf{X}^{(h) T}$. Here $\mathbf{X}^{(h)} \in \mathcal{R}^{|V| \times R}$ and $R \ll|V|$, so that $\mathbf{X}_{v}^{(h)}$ gives the location of node $v$ in some low-dimension space, and $\boldsymbol{S}^{(h)}$ is the proximity of all nodes. The number of parameters thus grows linearly, rather than quadratically with the number of vertices. In equations, we expand the upper plates in Figure 2(a) and (b), to get

$$
\boldsymbol{\theta}^{(h)}=f\left(\boldsymbol{X}^{(h)} \boldsymbol{X}^{(h) T}\right), \quad \mathbf{X}_{v}^{(h)} \sim N_{R}(\mathbf{0}, \mathbb{I}), v=1 \ldots, V
$$

Each population $y$ has a distribution over clusters $\boldsymbol{\beta}_{y}$. With prior probability half, the null hypothesis is true (we indicate this with the variable $\mathcal{T}$ ), in which case all populations have the same distribution $\boldsymbol{\beta}$. Otherwise, each population $g$ has its own distribution, $\boldsymbol{\beta}_{g}$. Thus,

$$
\begin{aligned}
\mathcal{T} \sim \operatorname{Bern}(1 / 2) \\
\text { If } \mathcal{T}=0: \quad \boldsymbol{\beta}_{1}=\ldots,=\boldsymbol{\beta}_{G} \sim \operatorname{Dir}(\alpha, \ldots, \alpha) \\
\quad \text { Else: } \quad \boldsymbol{\beta}_{g} \stackrel{i i d}{\sim} \operatorname{Dir}(\alpha, \ldots, \alpha) \text { for } g=1 \ldots G .
\end{aligned}
$$

Now, consider the case where each entity has only a single associated graph. Then the $n$th entity (belonging to population $Y_{n}$ ) has a graph $A_{n}$ distributed as

$$
C_{n}\left|Y_{n} \sim \boldsymbol{\beta}_{Y_{n}} \quad \mathbf{A}_{n}\right| C_{n} \sim F\left(\boldsymbol{\theta}^{\left(C_{n}\right)}\right)
$$

Here $C_{n}$ refers to the latent variable that identifies the cluster membership of entity $n$, which depends on the population entity $n$ is drawn from.

For the case where we have multiple network observations per entity, we add a layer to this hierarchical model. Now, each entity $n$ has their own distribution over clusters $\boldsymbol{\pi}_{n}$ centered around the population distribution:

$$
\boldsymbol{\pi}_{n} \sim \operatorname{Dir}\left(\beta_{Y_{n}}\right) .
$$

The graph $t$ of this entity is independently distributed as

$$
C_{n t}\left|Y_{n} \sim \boldsymbol{\pi}_{n}, \quad \mathbf{A}_{n t}\right| C_{n t} \sim F\left(\boldsymbol{\theta}^{\left(C_{n t}\right)}\right)
$$

Figure (2) summarizes our generative process for both cases.

\section{A. Model Inference}

We are given a set of network observations $\boldsymbol{A}$, each written as $A_{n t}$ where $n$ indexes entities and $t$, 'time'. For each $A_{n t}$, we are also given a population assignment $Y_{n} \in\{1,2\}$. Since we observe the population memberships $\boldsymbol{Y}$ and the networks $\boldsymbol{A}$, the inferential task is to learn $C_{n t}, \boldsymbol{\pi}_{n}, \boldsymbol{\beta}_{y}$ and $\boldsymbol{\theta}^{(h)}$. In the next section, we will use these variables as statistics in our hypothesis tests. For notational convenience, we will refer to a link between an arbitrary pair of nodes $i$ and $j$ with $l$, so that we can write $A_{n}[i, j]$ as $A_{n}[l]$. We also represent the weighted matrix with its vectorized lower triangular component $\mathcal{L}\left(\mathbf{A}_{n}\right)=\left(\mathcal{L}\left(A_{n t}\right)_{1}, \ldots, \mathcal{L}\left(A_{n t}\right)_{V(V-1) / 2}\right)$. For the general model specified above, we carry out posterior inference via a Gibbs sampler, whose individual updates we outline next:

1) Sample the cluster indicator for each graph.

This comes from the multinomial:

$$
\begin{aligned}
& P\left(C_{n t}=h \mid-\right)= \\
& \quad \frac{\pi_{n_{h}} \beta_{h} \prod_{l} P\left(\mathcal{L}\left(A_{n t}\right)_{l}=a_{l} \mid \theta_{l}^{(h)}\right)}{\sum_{m=1}^{H} \pi_{n_{m}} \beta_{m} \prod_{l} P\left(\mathcal{L}\left(A_{n t}\right)_{l}=a_{l} \mid \theta_{l}^{(m)}\right)}
\end{aligned}
$$

where $l \in[1, \ldots, V(V-1) / 2]$ and $a_{l} \mid \theta_{l}^{(h)} \sim$ $\left[F\left(\boldsymbol{\theta}^{(h)}\right)\right]_{l}$.

2) Sample the mixing probabilities for each entity $n$. With $\boldsymbol{m}_{n}$ a vector of cluster assignment counts of graphs of entity $n$

$$
\boldsymbol{\pi}_{n} \sim \operatorname{Dir}\left(\boldsymbol{\beta}_{Y_{n}}+\boldsymbol{m}_{n}\right)
$$

3) Sample the locations of the nodes for each cluster. With a Gaussian prior over the locations $\boldsymbol{X}_{n}$, and the weight-distribution parametrized after transforming through a link function $f$, this is a straightforward exercise in sampling from the posterior of a Gaussian with a nonlinear link function. Standard techniques exist to do this [15], [16], though we followed a recent idea involving the Polya-Gamma data-augmentation scheme [17].

4) Sample the testing indicator $\mathcal{T} \sim \operatorname{Bern}\left(P\left(\mathcal{H}_{1}\right) \mid-\right)$. Since $\mathcal{T}$ is the test-statistic central to our methodology, we discuss this in a bit more detail in the next section. The update rule is given by Equation 7.

5) Sample the mixing probabilities for each population $y$.

If $\mathcal{T}=0$ then for all $y, \boldsymbol{\beta}_{y}=\boldsymbol{\beta} \sim \operatorname{Dir}\left(\alpha+n_{1}, \ldots, \alpha+\right.$ $\left.n_{H}\right)$ where $n_{h}$ is the number of graphs in cluster $h$. If $\mathcal{T}=1$ then $\boldsymbol{\beta}_{y} \sim \operatorname{Dir}\left(\alpha+n_{1 y}, \ldots, \alpha+n_{H y}\right)$ for 


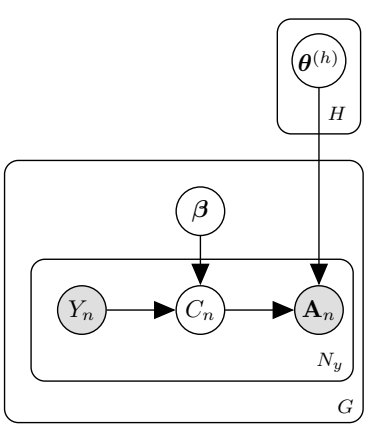

(A)

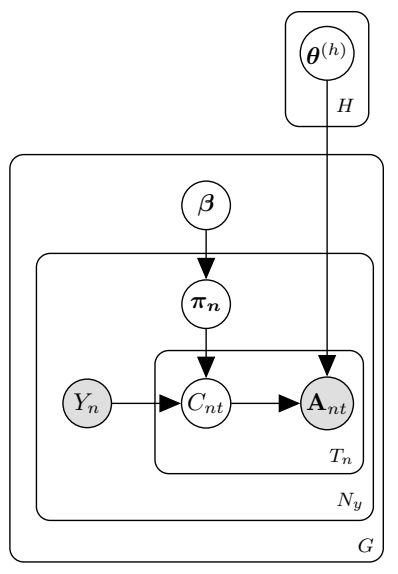

(B)
Figure 2: The graphical models are given by (A) fixed and (B) with time-varying structures.

each $y$, where $n_{i y}$ counts the number of graphs from population $y$ in cluster $i$.

\section{WEIGHTED-NETWORK COMPARISON TESTS}

For simplicity, we focus on the case where we have only two populations $(G=2)$. Under our formulation, the problem of hypothesis testing amounts to testing whether the population-level cluster assignment probabilities $\boldsymbol{\beta}_{1}$ and $\boldsymbol{\beta}_{2}$ are significantly different under the posterior. We elaborate on this below.

\section{A. Population-level network comparison test}

This task involves comparing the posterior probabilities of the two hypotheses, $H_{0}: \boldsymbol{\beta}_{1}=\boldsymbol{\beta}_{2}$ vs $H_{1}: \boldsymbol{\beta}_{1} \neq \boldsymbol{\beta}_{2}$. Since $H_{0}$ being true amounts to $\mathcal{T}=1$, our MCMC estimate of the probability equals the fraction of MCMC iterations where $\mathcal{T}=1$. We first describe how our Gibbs sampler updates this variable (step 4 of our Gibbs sampler). At any MCMC iteration, let $\boldsymbol{m}_{y}$ be the vector of cluster assignment counts for population $y$, with component $c$ giving the number of observations from population $y$ assigned to cluster $c: \boldsymbol{m}_{y}=$ $\left(\sum_{n ; y_{n}=y} \sum_{t} \mathbb{I}_{C_{n t}=1}, \ldots, \sum_{n ; y_{n}=y} \sum_{t} \mathbb{I}_{C_{n t}=H}\right)$. We write $\boldsymbol{m}=\boldsymbol{m}_{1}+\boldsymbol{m}_{2}$ (for the two populations in $G$, i.e., 1 and 2). Then, under the two hypotheses, these counts are distributed as

$$
\begin{aligned}
\mathcal{H}_{0}: & \boldsymbol{m}_{1}, \boldsymbol{m}_{2} \stackrel{i i d}{\sim} \operatorname{Mult}(\boldsymbol{\beta}), \\
& \boldsymbol{\beta} \sim \operatorname{Dirichlet}(\boldsymbol{\alpha}) \\
\mathcal{H}_{1}: & \boldsymbol{m}_{1} \sim \operatorname{Mult}\left(\boldsymbol{\beta}_{1}\right) \text { and } \boldsymbol{m}_{2} \sim \operatorname{Mult}\left(\boldsymbol{\beta}_{2}\right) \\
& \boldsymbol{\beta}_{\mathbf{1}}, \boldsymbol{\beta}_{\mathbf{2}} \sim \operatorname{Dirichlet}(\boldsymbol{\alpha})
\end{aligned}
$$

Marginalizing out the $\boldsymbol{\beta}^{\prime}$ s, and recalling that both hypotheses have the same prior probability, we can specify the posterior

$$
P\left(\mathcal{H}_{1} \mid-\right)=\frac{P\left(\boldsymbol{m}_{1} \mid \boldsymbol{\alpha}\right) P\left(\boldsymbol{m}_{2} \mid \boldsymbol{\alpha}\right)}{P(\boldsymbol{m} \mid \boldsymbol{\alpha})+P\left(\boldsymbol{m}_{1} \mid \boldsymbol{\alpha}\right) P\left(\boldsymbol{m}_{2} \mid \boldsymbol{\alpha}\right)}
$$

From the Dirichlet-multinomial conjugacy, we can write down the marginal probabilities of the observations, giving

$$
P\left(\mathcal{H}_{1} \mid-\right)=\frac{\prod_{y=1}^{2} \frac{B\left(\boldsymbol{\alpha}+\boldsymbol{m}_{y}\right)}{B(\boldsymbol{\alpha})}}{\frac{B(\boldsymbol{\alpha}+\boldsymbol{m})}{B(\boldsymbol{\alpha})}+\prod_{y=1}^{2} \frac{B\left(\boldsymbol{\alpha}+\boldsymbol{m}_{y}\right)}{B(\boldsymbol{\alpha})}}
$$

where $B(\cdot)$ is the multivariate beta function $B(x)=$ $\prod_{i=1}^{q} \frac{\Gamma\left(x_{i}\right)}{\Gamma\left(\sum_{i=1}^{q} x_{i}\right)}$. Every Gibbs iteration samples $\mathcal{T}$ from this, with the posterior probability of the alternative hypothesis, $P\left(\mathcal{H}_{1} \mid-\right)$, being the fraction of MCMC samples where $\mathcal{T}$ equals 1. If the estimate from Equation (7) is larger than a specified threshold (e.g., 0.95), we reject the null hypothesis and conclude that the populations are significantly different. We can use this network comparison (NC) test for both models in Figure 2. When we use the (fixed) model in 2 , we will refer to it as NC-F and when we use the (mixedmembership) model in $2 \mathrm{p}$, we will refer to it as NC-M.

\section{B. Entity-specific comparison test}

This task refers to do following hypothesis test: $H_{0}^{n_{1} n_{2}}$ : $\boldsymbol{\pi}_{n_{1}}=\boldsymbol{\pi}_{n_{2}}$ Vs $H_{1}^{n_{1} n_{2}}: \boldsymbol{\pi}_{n_{1}} \neq \boldsymbol{\pi}_{n_{2}}$ for any two users $n_{1}$ and $n_{2}$. Estimating this from our posterior samples is straightforward. Assuming multiple networks per entity, let $\tilde{\boldsymbol{m}}_{n}$ be a vector of counts for entity $n$, giving the number of observations assigned to each cluster. As mentioned earlier, the entity-specific distribution over clusters follows the distribution $\boldsymbol{\pi}_{n} \sim \operatorname{Dir}(\boldsymbol{\beta})$. Following the earlier logic, Equation 8 gives the posterior probability two given entities have different cluster assignment probabilities:

$$
P\left(\mathcal{H}_{1}^{n_{1} n_{2}} \mid-\right)=\frac{\prod_{i=1}^{2} \frac{B\left(\boldsymbol{\beta}+\tilde{\boldsymbol{m}}_{n_{i}}\right)}{B(\boldsymbol{\beta})}}{\frac{B(\boldsymbol{\beta}+\tilde{\boldsymbol{m}})}{B(\boldsymbol{\beta})}+\prod_{i=1}^{2} \frac{B\left(\boldsymbol{\beta}+\tilde{\boldsymbol{m}}_{n_{i}}\right)}{B(\boldsymbol{\beta})}}
$$

It is important to note that Equation 8 allows pairwise comparisons across populations, and therefore it is possible to have significantly similar entities from different populations and significantly different entities in the same population.

\section{Edge-specific comparison test}

This task refers to the following hypothesis test for an edge $l=(i, j), H_{0}^{l}: \boldsymbol{\theta}_{1}[l]=\boldsymbol{\theta}_{2}[l]$ vs $H_{1}^{l}: \boldsymbol{\theta}_{1}[l] \neq \boldsymbol{\theta}_{2}[l]$. For the edge application we use an adjusted version of Cramer's V-statistic proposed by [18] given by Equation 9 .

$$
p_{l}^{2}=\sum_{y=1}^{2} \mathbf{p}_{\mathcal{Y}} \sum_{a_{l}} \frac{P\left(\mathcal{L}(A)_{l}=a_{l} \mid \bar{\theta}_{y_{l}}\right)-P\left(\mathcal{L}(A)_{l}=a_{l} \mid \bar{\theta}_{l}\right)}{P\left(\mathcal{L}(A)_{l}=a_{l} \mid \bar{\theta}_{l}\right)}
$$

where $\mathbf{p}_{\mathcal{Y}}$ is the sample size proportion of each population, $\overline{\boldsymbol{\theta}}_{y}=\sum_{n=1}^{N} \sum_{h=1}^{H} \beta_{y h} \boldsymbol{\theta}^{(h)} \mathbb{I}_{y_{n}=y}$, and $\overline{\boldsymbol{\theta}}=\sum_{y=1}^{2} \frac{\overline{\boldsymbol{\theta}}_{y}}{2}$. If $p_{l} \approx 1$ then there is evidence that edge weights differ across the populations. 


\section{RELATED WORK}

Graph-based hypothesis testing and anomaly detection are topics of growing interest with diverse applications (see e.g., [6]). Many applications of hypothesis testing in network analysis focus on subgraphs within a larger graph (e.g., [7]), or one-sample tests comparing a single graph to a null model (e.g., [8]).

Work on populations of graphs can be divided on two areas: dynamic networks, in which one graph is replicated over time [19], [20] and [21]; and exchangeable graph modeling in which each graph is considered to be one observation for a single entity (see [3], [9], [10], [22] and [23]).

Our paper generalizes work from the latter category by allowing within-population heterogeneity, with each entity having multiple graphs with similar statistical properties. Both [9] and [3] deal with geometric characterizations of networks, and while their approaches are mathematically elegant, they are substantially less flexible than our work. [23] take a convolution neural network approach for nonaligned graphs, where there is no known mapping between nodes in each graph. This, coupled with the fact that their method requires the presense of node features, makes it unsuitable for our applications.

Most closely related to ours is the method presented in [10]. This method, which we will refer to DD, is a special case of our framework, where there is no within population variation, and where network edges are binary. For count or continuous-valued data, one might consider thresholding the edge weights of each entity and then applying the DD method. However this discards valuable information about the strength of the edge-weights, introduces sensitivity to threshold-level, and can reduce statistical power. Our method offers the ability to flexibly model such edge-weight information without any significant additional computational complexity. In particular, the computational time complexity of both our method and DD is $\mathcal{O}\left(N H R|V|^{2}\right)$ per iteration. Here $N$ is the number of networks, $H$ is the number of clusters, $R$ is the dimensionality of the low rank approximation, and $|V|$ is the number of nodes in each graph. In practice, $H$ and $R$ are small constants that do not grow with the data. In our experiments we compare with DD for different thresholds.

\section{EXPERIMENTS}

In order to assess the efficacy of our method, we divided our analysis into four parts: statistical power and typeI error analyses, population-level hypothesis tests, edgespecific hypothesis tests, and additional exploratory analysis.

We start with statistical power and type-I error analyses, the most important measures of assessing hypothesis tests. We investigate the efficacy of our (and competing) methods for varying sample sizes when the ground truth in known. We show that when the data are generated from a known two-population setup, our hypothesis testing framework produces significantly more accurate results and has lower variance, with respect to type-I error and statistical power, compared to a number of other baselines. We show that for time-varying data, the mixed membership extension of our model is essential for reliable inference. We also study the sensitivity of the method of [10] (which requires unweighted graphs) to threshold settings, for population-level hypothesis tests. We show that for heterogeneous data, the hypothesistest decisions are highly sensitive to threshold choice. We study the edge-specific hypothesis tests qualitatively, by visualizing the estimated model structure for each approach. We end by describing some additional insights that that our method gleans from the data. We start by describing the datasets.

\section{A. Datasets}

We generated synthetic weighted network data for two settings, the entity-homogeneous version from Figure $2 \mathrm{a}$ (NC-F, where each entity is represented by one graph) and the entity-heterogeneous version from Figure $2 \mathrm{p}$ (NC-M, where each entity is represented by multiple graphs). We also applied our framework to three real-world applications: a Twitter dataset from the political crisis in Brazil, two datasets about drugs usage on Instagram, and fMRI recordings of brains of human subjects.

Synthetic data (Homogeneous): We generate synthetic data from two populations whose underlying weight probability matrices $\boldsymbol{\theta}$ overlap around the middle set of nodes, but where population 1 has an elevated pattern of weight values for the first set of nodes, and population 2 in the final set. Figure 10 in Appendix VII-A shows this structure. We simulated 200 entities per population, with 100 nodes for each network. Given the structure, the weights of the edges were distributed according to a multivariate Zipf distribution [24]. See Appendix section VII-A for more details.

Synthetic data (Heteromogeneous): Using the same population structure as above, we also construct a time-varying dataset where each individual has four time points, resulting in four different graphs per entity. In this case, we have 50 entities and 100 nodes for each network. Given the dependency structure, the weights for each entity at each time point were distributed according to a multivariate Zipf distribution. Figure 11 in Appendix VII-A shows these structures. We use this dataset to compare the behavior of the NC-F and NC-M models under different scenarios.

\section{Real data:}

Twitter: Brazil has recently faced the worst economic/political crisis of its republic years. People were largely split into two sides: one who argued for the impeachment of the now former president, Dilma Rousseff, and the opposition who claimed that the process was a government coup. We crawled public Twitter posts from 

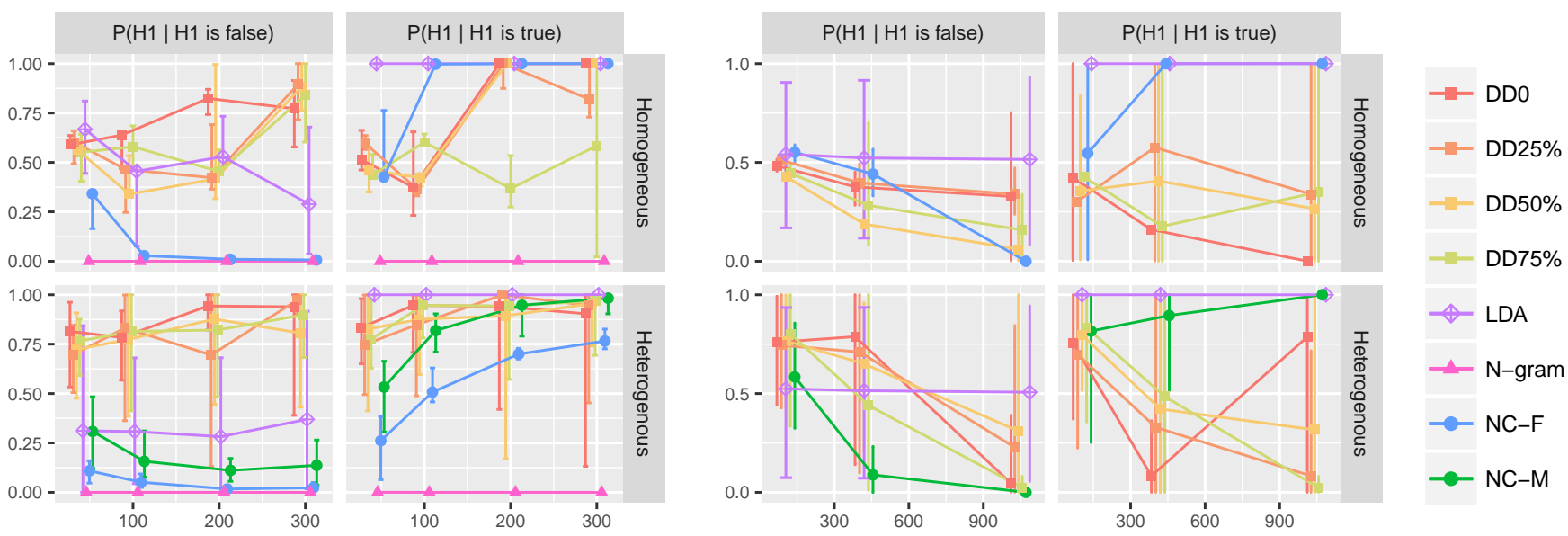

Figure 3: Type I error and statistical power curves for the synthetic (left) and twitter (right) data for increasing sample sizes

April 6th to May 31st 2016, using hashtags from both sides to collect tweets. The resulting dataset consists of 7,447 users (entities), 4, 233 for the proposition and 3,214 for opposition. In order to have appropriate data for the heterogeneous setting, we also split the dataset into time intervals, with each user having a network for every two weeks of tweets. We call this dataset "Twitter time". In this dataset, consisting of users with at least 15 days of tweets, we have a total of 2,098 users, 1,255 for proposition and 843 for opposition. Figure 1 shows sample co-occurrence networks from the two sides: Proposition and Opposition. Each edge-weight indicates the number of tweets of a user $n$ containing two words (nodes) in a time interval $t$.

Instagram: We collected public Instagram comments with hashtags referring to the two most common drugs to treat ADHD (Adderall and Ritalin) and Depression (Prozac and Zoloft). These medications all have additional uses (and consequently symptoms), for instance, Adderall is known for loss of appetite, and as an aid for academic performance. Our dataset consists of 65 users with 44, 408 posts for \#adderall, 21 with 17, 466 for \#ritalin, 111 with 129, 405 for \#prozac, and 35 with 29,357 for \#zoloft.

fMRI brain images: Functional magnetic resonance imaging (fMRI) captures activity in the brain by measuring blood flow from one region of the brain to another. We used the MRN-111 datase 17 which consists of functional magnetic resonance images (fMRI) for 114 subjects (entities). As in [25] we used a total of 68 brain regions, 34 from the left hemisphere and 34 from the right. Nodes represent brain regions, and weights, white matter density across nodes. We compare brain activity across characteristics like Sex (Male vs Female), and personality traits like creative level ( $\leq 90$ vs $\geq 111$ ), extroversion ( $\leq 30$ vs $\geq 35$ ). Values for creative level (CCI) and extroversion are given from a psy-

\footnotetext{
1http://openconnecto.me/data/public/MR/
}

chometric scale determined by the corresponding scientific literature, those thresholds were chosen to illustrate a clear LowVsHigh setting. Figure 1 shows sample brain networks of the MRN111 dataset for female and male individuals. We observe significant variability in these weights, suggesting that thresholding can lead to loss of information.

\section{B. Baselines}

We compared our NC-F and NC-M methods with the following baselines:

1) LDA (topic modeling) [13]: This treats each entity as a document made up of 'topics' (each corresponding to a distribution over word-count patterns).

2) N-gram language model [14]: We use observed bigrams frequencies to estimate co-occurrence probabilities.

3) DD network model [10]: As stated previously, DD forms a special instance of our more general framework for unweighted networks. In order to apply DD, we need first threshold the weighted network observations. We do so using the following criterion [26]:

$$
p_{i j}=\frac{\text { co-occurances between words } i \text { and } j}{\min (\text { counts of words } i \text { and } j)}
$$

Then $A_{n}[i, j]=1$ if $p_{i j}>$ threshold for a chosen threshold level.

Since N-gram and LDA do not directly allow us to estimate $P\left(H_{1}\right)$, we use a Kolmogorov-Smirnov test on the words distribution to perform an overall hypothesis test between populations for the $\mathrm{N}$-gram model, and a chi-square test for topic assignments across populations for LDA.

\section{Hyperparameters tunning}

DD and our method require setting the number of clusters $H$, the dimensionality of the low-rank factorization $R$, 
the Dirichlet concentration parameters $\alpha$ for $\boldsymbol{\beta}$, and the prior probability of $P\left(H_{1}\right)$. For our experiments, we fixed $H=15, R=10, \alpha=1 / H$ and $P\left(H_{1}\right)=0.5$. We found that $H=15$ was more than enough clusters for all instances, larger numbers resulting in empty clusters. Most of our experiments focus on settings with count-valued weights, and in the case of word co-occurrences, the weight between words $i$ and $j$ is bounded by the smaller of the number of occurrences of the two words $i$ and $j$. For this setting, we therefore used the logit link and the binomial likelihood. On the other hand, the Brain dataset has count-valued weights with unbounded support, and we used Exponential link and the Poisson likelihood. Our results were fairly robust to hyperparameters settings. For our MCMC algorithm, we observed good mixing properties, and used 1300 Gibbs samples with an extra 200 burn-in samples.

For LDA and N-Gram, we used settings following implementations from [27] and [28], respectively.

\section{Results}

Type-I error and statistical power: Type-I errors or false positives arise when a model incorrectly marks two populations as different when actually the null hypothesis is true, i.e., $P\left(H_{1} \mid H_{1}\right.$ is false $)$. Ideally, type-I error rates should be 0.05 or less. Statistical power shows if the models can correctly determine when the populations are different, and $P\left(H_{1} \mid H_{1}\right.$ is true) should be close to 1 . Measuring these quantities requires access to ground truth. For the Brazil dataset, the disparity of political tendencies betweer opposition and proposition is clear enough that we treat $i$ as ground truth (For the other real datasets, we do not have ground truth available).

We consider four sample sizes for the synthetic data: 50 100, 200 and 300. For the twitter data, we consider thre sample sizes: 105 ( 5\%), $420(\sim 20 \%)$ and $1049(\sim 50 \%)$ For DD which requires thresholding, we used four differen threshold-levels, 0, 25\%, 50\% and $75 \%$. For all methods we compute $P\left(H_{1} \mid-\right)$ under different settings. In order tc estimate variance $P\left(H_{1} \mid-\right)$, we generated 20 datasets for each sample size.

Figure 3 presents the Type-I error (i.e., $P\left(H_{1} \mid H_{1}\right.$ is false)) and power curves (i.e., $P\left(H_{1} \mid H_{1}\right.$ is true) ) for increasing sample sizes for each method for synthetic data (left) and real world data (right). Each data point shown is the mean of the 20 trials for the respective sample size, we also present the range of $5 \%$ to $95 \%$ percentiles. We see that NC-F has the best overall performance both when $H_{1}$ is true and for $H_{1}$ false in the homogeneous scenario. $\mathrm{N}$-gram has the worst performance overall so we do not consider this baseline in the real data. LDA have a good overall power, however it performs poorly as far as Type I error goes, with the largest variance. Further, LDA is not able to capture probabilistic structure underlying the data (we discuss this later). DD's performance is not so good for Type I error in the synthetic data, but it is good overall for power. This is not the case for the real data though, where power is poor and varies significantly with threshold. This sensitivity to thresholdlevel confirms the original motivation for this work. NC$\mathrm{M}$ outperformed all other methods for the heterogeneous data, and NC-F was the second best even though it does not account for heterogeneity. This is due to the fact that our method accounts for the weight distributions, and thus can handle overdispersed counts relatively well.

We also investigate how the number of active users in the dataset affects the overall power performance. First, we define "activeness" as a function of number of days a user tweeted. We created datasets restricting to users with at least 2, 5 and 10 days of tweets. Thus, we assume that in the dataset with users having at least 10 days of tweets, there are a significantly larger number of active users than in dataset with a minimum of 2 days of tweets. Note that we are assessing only statistical power here, therefore we are only looking to the case that $H_{1}$ is true, i.e., "PropositionVsOpposition". Figure 4 shows the power curve for each these datasets. As expected, our method needs fewer observations to find statistical difference between populations with more active users in the dataset, in other words NC-F has the ability to differentiate between populations easier as the proportion of active users increases. DD does not improve with larger sample size which suggests DD loses the ability to determine whether populations differ in a higher heterogeneous setting.

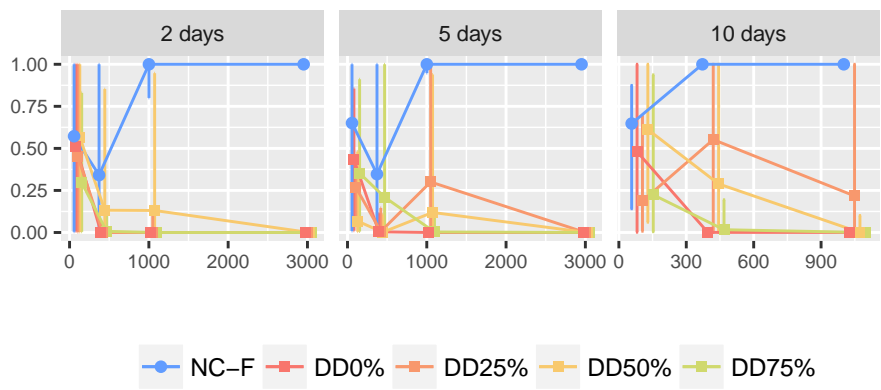

Figure 4: Power curves of NC-F and DD (multiple threshold levels) for increasing proportion of active users

Population-level hypothesis test: In the previous results, we had a glimpse of the sensibility of threshold choice in terms of decision making on the hypothesis testing procedure. Here, we aim to analyze this further. We estimate the posterior probability of $H_{1}$ for all observations of all the datasets. We compare our results with DD for 10 different threshold levels $(0,10 \%, 20 \%, \ldots, 90 \%)$. Note that NC-F and NC-M do not vary with threshold level. Here, in addition to the Twitter data, we include results testing the fMRI datasetcomparing populations based on the creative index (CCI). [10] found a significant difference in Brain connectivity between non creative individuals $(C C I \leq 90)$ vs creative 
subjects $(C C I \geq 111)$. In their tests, the graphs were thresholded at 0 .

Figure 5 shows DD represented as red solid squares, NC-F and NC-M as blue and green lines, respectively. Again, DD exhibits sensitivity to the threshold choice making inferences unreliable. For instance, if we consider testing whether populations Proposition and Opposition are significantly different and use a $60 \%$ threshold for Twitter time, we would reject the null, since the posterior of $P\left(H_{1}\right) \approx 1$. However, if we slightly change the threshold level to $50 \%$, $P\left(H_{1}\right) \approx 0.5$ meaning that there is not enough evidence to support that they are statistically different and we accept the null. The same behavior can be seen on the fMRI dataset where the threshold at 0 is statistical significant for LowVsHigh, however it is not for any other threshold. Overall, we found that our methods NC-M and NC-F are more reliable, since they avoid the need for practitioners to make sensitive preprocessing choices.

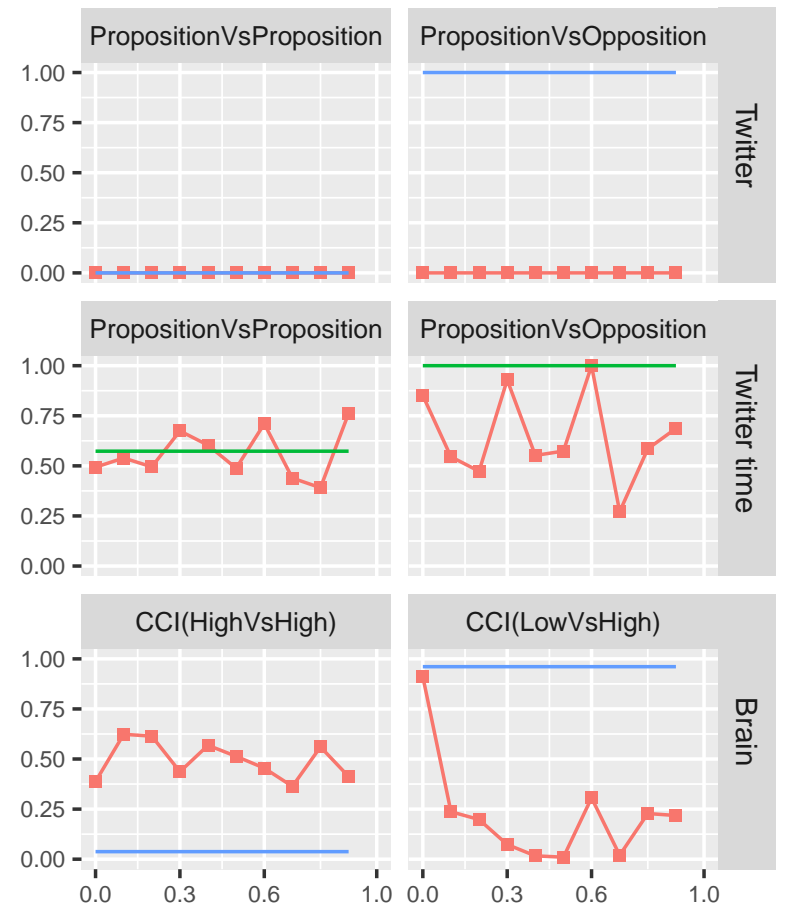

Figure 5: $P\left(H_{1} \mid-\right)$ across threshold levels for three datasets for when $H_{1}$ is false (left column) and $H_{1}$ is true (right column). NC-F and NC-M are represented as blue and green lines, respectively.

Edge-specific level hypothesis test: Another important task is that of retrieving the structure of the co-occurrences probabilities. For better visualization, we generated a version of the synthetic homogeneous with 20 nodes, and we look at differences between true and predicted edge probability matrices for both populations, i.e. we compute the estimated difference $\overline{\boldsymbol{\theta}}_{1}-\overline{\boldsymbol{\theta}}_{2}$ for each model and compared with the ground truth. In Figure 6, we see that our proposed framework accurately recovers the structure of the ground truth. The DD0 also retrieves the structure of population 1, however it performs poorly for population 2 . This is related to the sensitivity of results to the threshold-level, suggesting this needs to be chosen carefully across different scenarios. Our models NC-F and NC-M both do not require such handtuning, and further exploit values of the pre-thresholded counts for more accurate inference. Unsurprisingly, all the other models fail to correctly learn the structure used to simulate the data.

For the Twitter and Instagram datasets, we looked at each edge, and identified those that are different using a 0.1 significance level. Figure 7 shows that the NC-F model was able to capture a clear pattern of significantly different use of words among populations for the Brazil dataset, as opposed to the other modeling schemes which look almost random. For the Instagram drug data, the edge-specific hypothesis testing matrix structure is much more significant compared with the Twitter case. One reason for this is that there is a group of Ritalin users that are German, and their words differ from others.

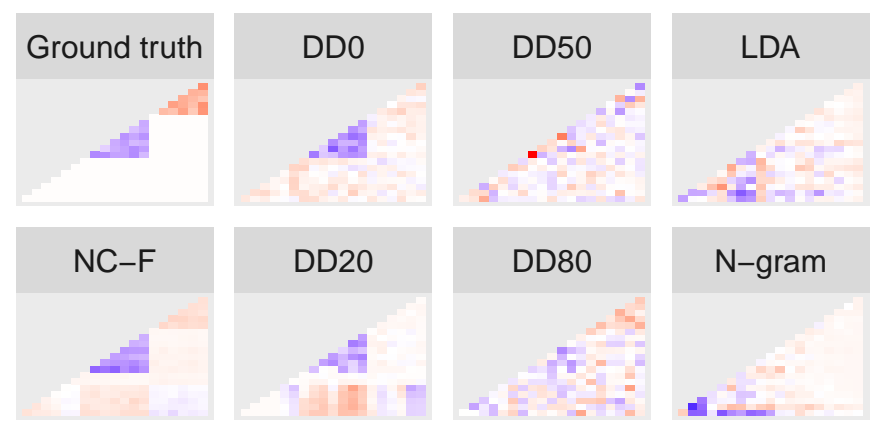

Figure 6: Edge-specific probabilities difference

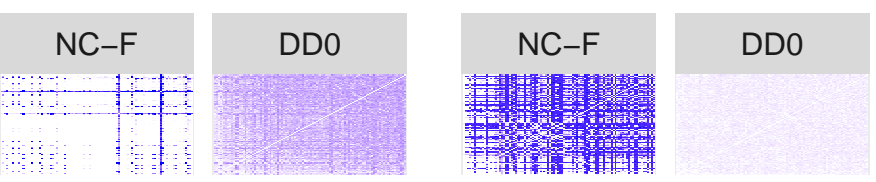

Figure 7: Edge-specific tests for Twitter (left) and Instagram (right).

Exploratory analysis: Here, we show some additional insights that our methods are capable of capturing. One interesting fact of the Brazil political scenario is that many high frequency words were extensively used across both populations, examples being "impeachmentja" (impeachment now), "lavajato" (carwash), "golpenao" (no coup), "direitos" 
(rights). However, using the probability structure $\overline{\boldsymbol{\theta}}$ estimated from our framework, we can make some interesting insights about how the two sides frame the issues differently. Figure 8 plots the difference of the link probability for each high frequency word used in conjunction (co-occurrence) with all other words, across the two sides-if the value is larger than zero then it is a 'proposition expression', otherwise it is an 'opposition expression'. For instance, "lavajato" is the name of the investigation and if it is used with "motivo" (motive) is a proposition statement where if it is used with "luta" (fight) then it is a clear opposition one. From Figure 8 it is clear that the two sides use sets of words (e.g., phrases) quite differently.

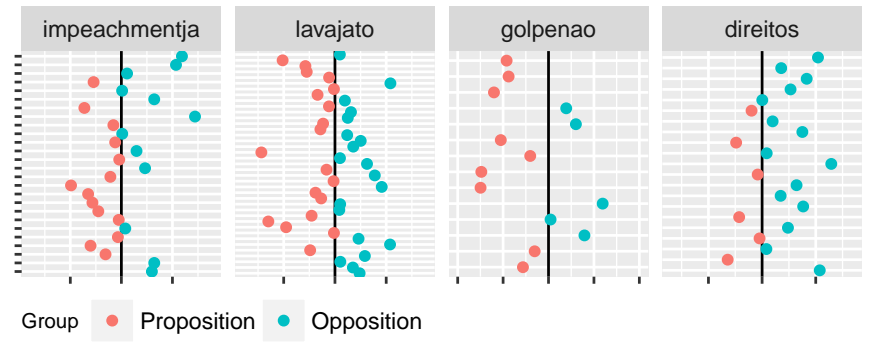

Figure 8: Swing words: differences in edge probabilities for example high frequency terms.

Figure 9 presents additional results for the Instagram and fMRI datasets. In this case, we look again to the behavior of $P\left(H_{1} \mid-\right)$ across threshold levels. It is important to highlight that we do not have a ground truth information to compare our findings with, however it is an additional set of results to explore the assessment of significant difference between two populations, and to note the lack of robustness of DD wrt threshold choice.

\section{CONCLUSION}

This paper presents the first steps towards routine and systematic hypothesis testing on populations of weighted networks. Our statistical framework applies both to settings where entities from each population have single graphs associated with them, as well as settings where each entity has associated a set of graphs (we call these without and with within-population heterogeneity). Through a flexible and general clustering mechanism for replicated weighted networks, our framework offers a powerful and accurate hypothesis testing at three levels: population-level, entityspecific and edge-specific. We applied our model to study communication behavior on real social media data (Instagram and Twitter), as well as for brain connectivity data. We saw that by not relying on a user-specified threshold, our proposed method offers robustness over the methodology of [10], besides outperforming other baselines like LDA, Ngram language models.

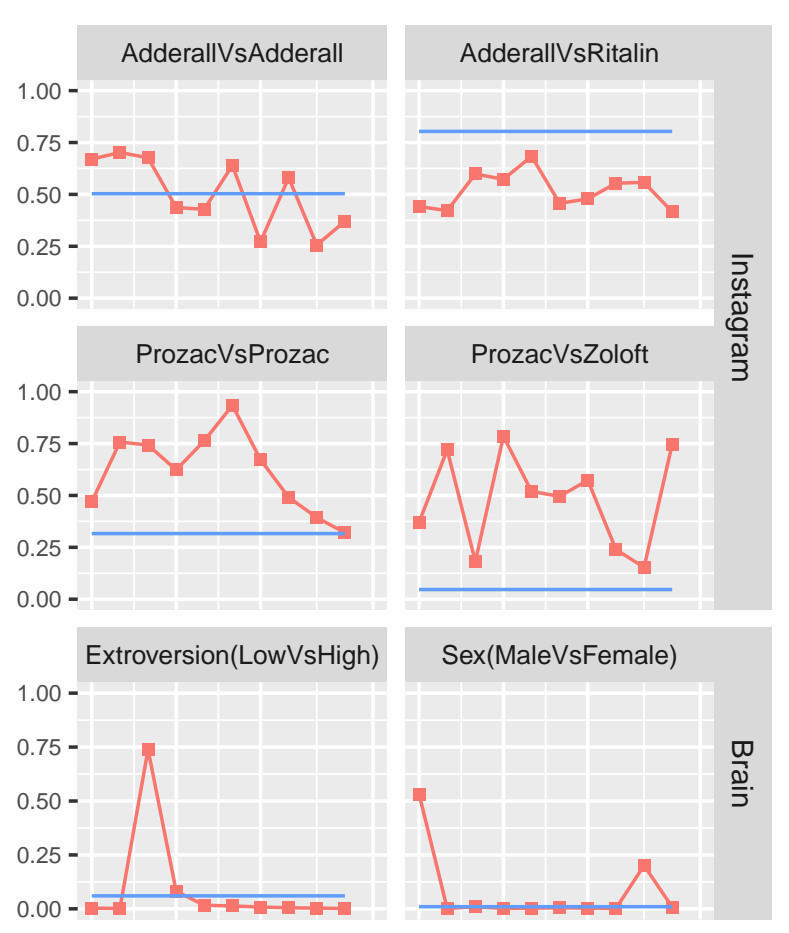

Figure 9: $P\left(H_{1} \mid-\right)$ across threshold levels for Instagram and fMRI datasets.

\section{REFERENCES}

[1] G. Gomes, V. Rao, and J. Neville, "Multi-level hypothesis testing for populations of heterogeneous networks," in Data Mining (ICDM), 2018 IEEE 18th International Conference on Data Mining. IEEE, 2018.

[2] G. Li, M. Semerci, B. Yener, and M. J. Zaki, "Graph classification via topological and label attributes," in Proceedings of the 9th international workshop on mining and learning with graphs (MLG), San Diego, USA, vol. 2, 2011.

[3] D. M. Asta and C. R. Shalizi, "Geometric network comparisons," in Proceedings of the Thirty-First Conference on Uncertainty in Artificial Intelligence. AUAI Press, 2015, pp. 102-110.

[4] S. S. Mukherjee, P. Sarkar, and L. Lin, "On clustering network-valued data," Advances in Neural Information Processing Systems, pp. 7074-7084, 2017.

[5] F. Arrigo, P. Grindrod, D. J. Higham, and V. Noferini, "On the exponential generating function for non-backtracking walks," Linear Algebra and its Applications, vol. 556, pp. 381-399, 2018.

[6] L. Akoglu, H. Tong, and D. Koutra, "Graph based anomaly detection and description: a survey," Data Mining and Knowledge Discovery, vol. 29, no. 3, pp. 626-688, 2015.

[7] M. Mongiov1, P. Bogdanov, R. Ranca, E. Papalexakis, C. Faloutsos, and A. Singh, "Netspot: Spotting significant anomalous regions on dynamic networks," in SIAM International Conference on Data Mining, 2013. 
[8] S. Moreno and J. Neville, "Network hypothesis testing using mixed kronecker product graph models," International Conference on Data Mining, 2013.

[9] C. E. Ginestet, J. Li, P. Balachandran, S. Rosenberg, E. D. Kolaczyk et al., "Hypothesis testing for network data in functional neuroimaging," The Annals of Applied Statistics, vol. 11, no. 2, pp. 725-750, 2017.

[10] D. Durante, D. B. Dunson et al., "Bayesian inference and testing of group differences in brain networks," Bayesian Analysis, vol. 13, no. 1, pp. 29-58, 2018.

[11] T. P. Peixoto, "Inferring the mesoscale structure of layered, edge-valued, and time-varying networks," Physical Review E, vol. 92, no. 4, p. 042807, 2015.

[12] R. B. Correia, L. Li, and L. M. Rocha, "Monitoring potential drug interactions and reactions via network analysis of instagram user timelines," in Pacific Symposium on Biocomputing. Pacific Symposium on Biocomputing, vol. 21. NIH Public Access, 2016, p. 492.

[13] D. M. Blei, A. Y. Ng, and M. I. Jordan, "Latent dirichlet allocation," Journal of machine Learning research, vol. 3, no. Jan, pp. 993-1022, 2003.

[14] S. Katz, "Estimation of probabilities from sparse data for the language model component of a speech recognizer," IEEE transactions on acoustics, speech, and signal processing, vol. 35, no. 3, pp. 400-401, 1987.

[15] S. Duane, A. D. Kennedy, B. J. Pendleton, and D. Roweth, "Hybrid monte carlo," Physics letters B, vol. 195, no. 2, pp. 216-222, 1987.

[16] I. Murray, R. P. Adams, and D. J. MacKay, "Elliptical slice sampling," J. Mach. Learn. Res. W\&CP, vol. 9, 2010.

[17] N. G. Polson, J. G. Scott, and J. Windle, "Bayesian inference for logistic models using pólya-gamma latent variables," Journal of the American statistical Association, vol. 108, no. 504, pp. 1339-1349, 2013.

[18] D. B. Dunson and C. Xing, "Nonparametric bayes modeling of multivariate categorical data," Journal of the American Statistical Association, vol. 104, no. 487, pp. 1042-1051, 2009.

[19] P. Sarkar, D. Chakrabarti, and M. Jordan, "Nonparametric link prediction in dynamic networks," arXiv preprint arXiv:1206.6394, 2012.

[20] L. Charlin, R. Ranganath, J. McInerney, and D. M. Blei, "Dynamic poisson factorization," in Proceedings of the 9th ACM Conference on Recommender Systems. ACM, 2015, pp. $155-162$.

[21] D. Durante and D. B. Dunson, "Nonparametric bayes dynamic modelling of relational data," Biometrika, pp. 1-16, 2014.

[22] D. Durante, D. B. Dunson, and J. T. Vogelstein, "Nonparametric bayes modeling of populations of networks," Journal of the American Statistical Association, 2015.

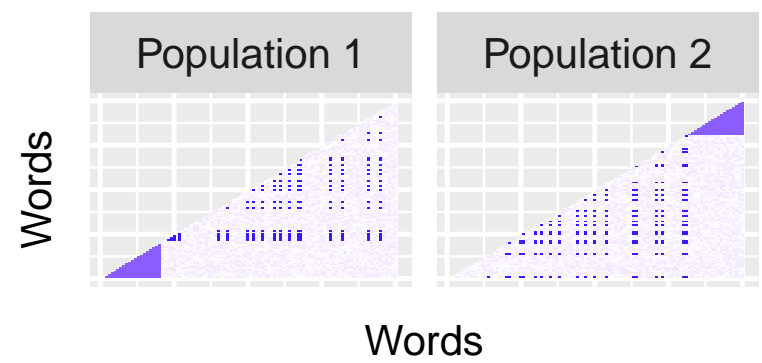

Figure 10: Structure used to simulate homogeneous data

[23] D. K. Duvenaud, D. Maclaurin, J. Iparraguirre, R. Bombarell, T. Hirzel, A. Aspuru-Guzik, and R. P. Adams, "Convolutional networks on graphs for learning molecular fingerprints," in Advances in neural information processing systems, 2015, pp. 2224-2232.

[24] H.-C. Yeh, "Six multivariate zipf distributions and their related properties," Statistics \& probability letters, vol. 56, no. 2, pp. 131-141, 2002.

[25] R. S. Desikan, F. Ségonne, B. Fischl, B. T. Quinn, B. C. Dickerson, D. Blacker, R. L. Buckner, A. M. Dale, R. P. Maguire, B. T. Hyman et al., "An automated labeling system for subdividing the human cerebral cortex on mri scans into gyral based regions of interest," Neuroimage, vol. 31, no. 3, pp. 968-980, 2006.

[26] T. Simas and L. M. Rocha, "Distance closures on complex networks," Network Science, vol. 3, no. 2, pp. 227-268, 2015.

[27] R. Řehůřrek and P. Sojka, "Software Framework for Topic Modelling with Large Corpora," in Proceedings of the LREC 2010 Workshop on New Challenges for NLP Frameworks. Valletta, Malta: ELRA, May 2010, pp. 45-50, http://is.muni. cz/publication/884893/en

[28] D. Schmidt and C. Heckendorf, "ngram: Fast n-gram tokenization," 2017, R package version 3.0.4. [Online]. Available: https://cran.r-project.org/package=ngram

[29] G. K. Zipf, Human behavior and the principle of least effort. Addison-Wesley Press, 1949.

\section{APPENDIX}

\section{A. Synthetic data details}

The synthetic data was generated to have the same construction of the Twitter dataset set, i.e. a set word cooccurrences networks. In this sense, each node is a word and each edge is a co-occurrence of two words. Moreover, each pair of words can co-occur at most the minimum occurrence of each individual word. In other words, say words $i$ and $j$ occurred $x$ and $y$ times, respectively, then the co-occurrence of words $i$ and $j$ is at $\operatorname{most} \min (x, y)$. Hence, in order to generate graphs in this setting, we need to have the individual occurrences of all the words and probability structure for the co-occurrences. Since Zipf's law [29] , or 


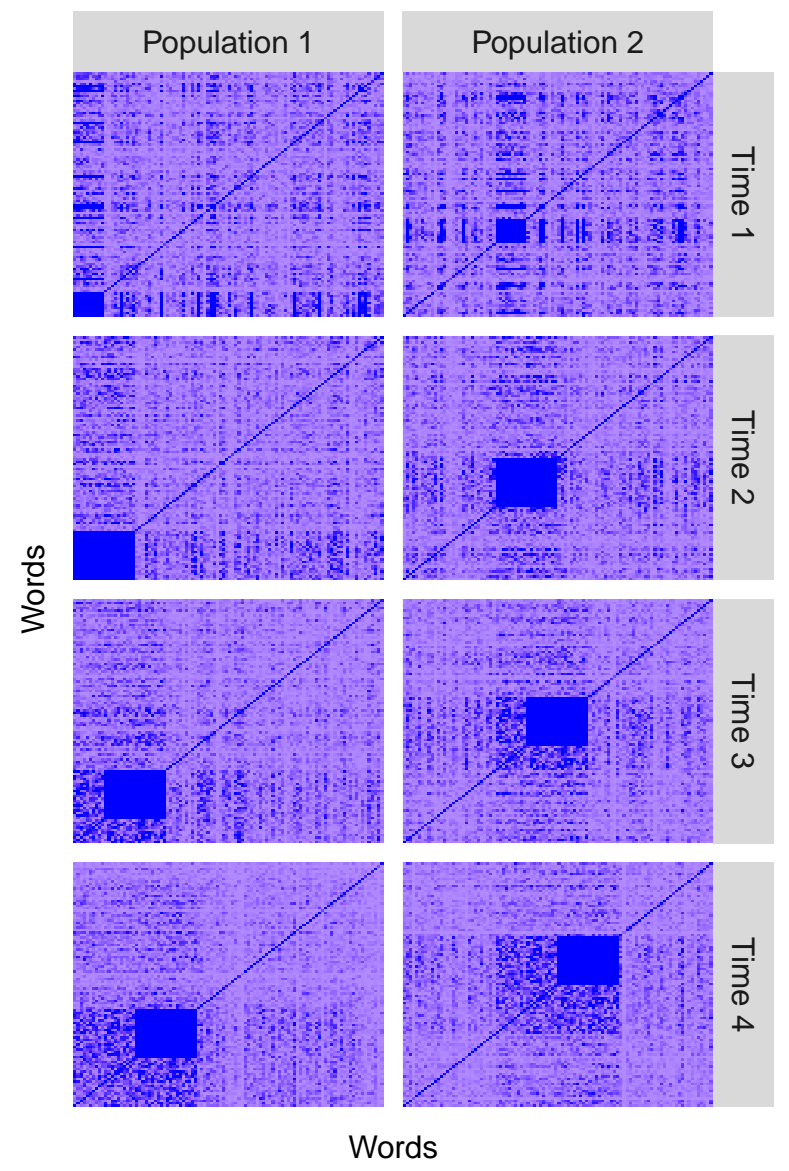

Figure 11: Structure used to simulate heterogeneous data

discrete Pareto, is almost always used to describe words frequencies, we used a multivariate Zipf generating process [24] to generate the individual counts. Equation 10 shows how to generate multivariate Zipf's values, $\boldsymbol{X}_{n} \in \mathbb{R}^{V}$ is the vector with all the individual counts of $V$ words for entity $n$. In our case, we considered the standard Zipf where $\lambda=1$.

$$
\begin{aligned}
& p \sim \operatorname{Beta}(1, \lambda) \\
& \boldsymbol{X}_{n} \mid p \stackrel{\text { ind }}{\sim} \operatorname{Geo}(p)
\end{aligned} \Rightarrow \boldsymbol{X}_{n} \sim M^{(m)} \operatorname{Zipf}(I V)(0, \lambda, 1,1)
$$

The structures were arbitrarily chosen to have a clear difference across populations. Figure 10 shows the structure used to simulate the homogeneous data used on the experiments section and Figure 11 shows the structure used to simulate for the heterogeneous data. Given the individual counts and the probability of each edge, we simulate edge count using Binomial distribution. Formally, $A_{n i j} \sim$ $\operatorname{Bin}\left(\min \left(X_{n i}, X_{n j}\right), \boldsymbol{\theta}_{i j}\right)$, where $A_{n i j}$ is the co-occurrence of words $i$ and $j, X_{n i}$ and $X_{n j}$ are their individual counts and $\boldsymbol{\theta}_{i j}$ is the probability of $i$ and $j$ co-occur once. 